\title{
A rare case of panhypopituitarism: \\ Pituitary stalk interruption syndrome
}

Kamil BASKOY ${ }^{1}$, Seyid Ahmet AY ${ }^{1}$, Barbaros Başbuğ ${ }^{2}$, Ferhat DENIZ ${ }^{1}$, Arif YONEM ${ }^{1}$

1.Dep. of Endocrinology, Haydarpaşa Training Hospital, GATA; 2.Dep. of Internal Medicine, Haydarpaşa Training Hospital, GATA, Istanbul/TURKEY

Introduction: Hypopituitarism is characterized by decreasing of pituitary hormones, which can result from diseases of the pituitary gland or the hypothalamus. The prevalence of hypopituitarism is $30-45$ out of 100,000 in the world wide. Causes of panhypopituitarism are non-tumoral hypotalomopituitary reasons (\%50), pituitary tumors (\%43) and extra-pituitary tumors (\%7). Here we want to present a very rare case of pan hypopituitarism; Pituitary stalk interruption syndrome(PSIS).

Case report: A 22-year-old male patient applied to our policlinic for routine control while continuing His medical treatments for panhypopituitarism. His ongoing replacement therapy included hydrocortisone, levothyroxine and testosterone hormones for 10 years. His height was $1.85 \mathrm{~cm}$, weight $75 \mathrm{~kg}$, and had a pale appearance on the face. Laboratory examination results at our outpatient clinic were compatible with panhypopituitarism. MR imaging was done for panhypopituitarism etiology. Adenohypophysis and infundibulum were absenton MR screening. Neurohypophysis was located at infundibular recess neighborhood in front of third. ventricle. As confirmed at MRI, the reason of panhypopituitarism was Pituitary stalk interruption syndromeWe were not able to do molecular research due to our laboratory insuffiency. His drug regime was reviewed and advised to come regular follows.

Conclusion: Congenital panhypopituitarism is often related with a small pituitary, thinned or interrupted stalk, and ectopic neurohypophysis, all together referred the Pituitary stalk interruption syndrome (PSIS). Especially due to improvements in screening, the number of PSIS diagnosis has increased. Perinatal events and genetic mutations are accused by at PSIS etiology but exact reason hasn't illuminated yet. Usually posterior hypophysis functions are protected at PSIS, but, different degree impairment are seen in anterior hypophysis functions. In this case, especially all anterior hypophysis hormones were deteriorated thought neuroyhypophysis hormones were normal. Patient was clinically benefited from hormone replacement treatments. 